\title{
Everything Old is New Again
}

\author{
Anita Florendo-Cumbermack, Dan Selchen, Sarah A. Morrow, Manas Sharma, David Steven, \\ Lee C. Ang, Courtney Casserly, Jorge Burneo, Marcelo Kremenchutzky, Robert Hammond
}

Keywords: demyelination, immune system, inflammatory diseases, multiple sclerosis, multiple sclerosis MRI, neuropathology

\section{Case Presentation: Dr. Florendo-Cumbermack}

\section{Initial Presentation to the Emergency Department}

A 62-year-old woman was brought by ambulance to the emergency department (ED) with confusion and right-sided weakness. Upon initial assessment by the ED physician, a code stroke was called and she was assessed by the neurology team. The only information available at that time was that she was staying with her daughter and was found outside her daughter's home confused and with right-sided weakness.

She was awake but nonverbal and unable to obey commands. Her head and eyes were deviated to the right and eyes did not cross the midline even with vestibulo-ocular testing. Visual field testing by blink to threat was inconsistent. There was a right lower facial droop and minimal movement of the right arm and leg. She did not cooperate with testing for ataxia and sensory testing was inconsistent. Assessment of neglect was also difficult.

\section{Discussion: Dr. SELCHEN}

The history provided suggests extensive involvement of the left hemisphere.

If this was an acute hemispheric stroke, one would expect the eyes to be deviated to the left-towards the side of the lesion. The presence of eye deviation towards the right is not in keeping with a left hemispheric stroke. There is a phenomenon known as "wrongway eyes" that would most commonly be associated with seizure activity in the left hemisphere, involving the left frontal eye field area causing the eyes to be pushed to the right. A thalamic lesion such as a haemorrhage could cause contralateral hemiparesis, aphasia, and wrong-way eyes, but this would be very rare. Another possibility would be a lesion in the left pons causing contralateral weakness and rightward eye deviation. This would not, however, explain the aphasia unless there were multiple acute lesions.

Pending further history, a seizure related to a left frontal focus is the probable explanation.

\section{Case Presentation: Dr. Florendo-Cumbermack}

Just before her computed tomography (CT) imaging, she had two seizures starting with right-sided tonic movements and then generalized tonic-clonic activity. The seizures lasted less than a minute each and occurred in close succession. She was given $2 \mathrm{mg}$ of lorazepam and loaded with $1 \mathrm{~g}$ of phenytoin. She then went for a CT scan of the head.
Her family members subsequently arrived and were able to provide additional history. Her daughter confirmed that she found the patient outside the home, confused with right-sided weakness. She noticed tonic-clonic movements of the right limbs twice before arrival of the EMS.

Relatives also indicated that 11 years ago, she had presented to another institution with right-sided weakness, behavioural changes, disorientation, and visual hallucinations. Investigations at that time included magnetic resonance imaging (MRI) of the head that showed a left hemispheric mass lesion and a diagnostic procedure was performed. Family confirmed that with medical treatment, the patient improved but was unable to return to work because of ongoing right upper extremity weakness. She was, however, independent with activities of daily living and independent activities of daily living.

The patient remained free of new symptoms until 6 months before her ED presentation, when she began to experience progressive right-sided weakness and numbness. She had a number of recent falls that led to the use of a cane. She also complained of headaches and her family noted "mood swings." She was seen in the local ED on multiple occasions for the falls.

Three months ago, she started bumping into objects on her right side. She had no specific visual complaints. She also developed difficulty with her memory, writing, and other tasks such as brushing her teeth.

She had been otherwise well. She had a 35 pack-year cigarette smoking history. She was on citalopram $20 \mathrm{mg}$ daily. Her mother had coronary artery disease. One of her two daughters had a uterine carcinoma with metastases to the brain and two of her maternal first cousins had multiple sclerosis (MS).

The day after admission, she was awake and alert. Her speech was non-fluent with impaired naming and repetition. Her comprehension was intact for simple commands. Further cognitive assessment was difficult. She had mild right upper motor neuron type facial weakness and a complete right homonymous hemianopia. The tone in her arms was normal but she had a spastic

From the Schulich School of Medicine \& Dentistry, Western University, London, Ontario, Canada (AF-C, SAM, MS, DS, LCA, CC, JB, MK, RH); London Health Sciences Centre, London, Ontario, Canada (AF-C, SAM, Q2 MS, DS, LCA, CC, JB, MK, RH); University of Toronto, Toronto, Ontario, Canada (DS, CC); St. Michael's Hospital, Toronto, Ontario, Canada (DS, CC).

Received December 4, 2014. Final Revisions Submitted May 10, 2015. Correspondence to: Robert Hammond, Rm A3-148, Department of Pathology, LHSC-UH, 339 Windermere Road, London, ON N6A 5A5, Canada.

Email: RobertHammond@lhsc.on.ca. 
catch at the right knee. Power was full on the left but Medical Research Council grade 3-4/5 in the right limbs in a pyramidal distribution. Deep tendon reflexes were brisk on the right, with a right upgoing plantar response. Pin and vibration sensation were intact, but sensory extinction was noted on the right to double simultaneous stimulation. There was no appendicular ataxia and her systemic examination was unremarkable.

\section{DisCuSSION: DR. SELCHEN}

There seems to be extensive involvement of the left hemisphere, primarily parietal and frontal. The apparent recurrence after an 11-year hiatus with a preceding progressive story leaves few options.

An ischemic process is again unlikely in this case. The presentation is highly atypical for vasculitis because of the 11-year gap. Another consideration would be a vascular malformation with recurrent bleeds but this would have been immediately apparent from a CT scan and does not fit with the pattern of the current history. A mitochondrial disorder could also be considered, but the patient's age and the recent progressive picture make this a remote possibility. The tempo of the clinical presentation would be out of keeping for a tumour. Recurrent abscess is a very remote possibility.

The most likely etiology would therefore be an inflammatory/ demyelinating process. Acute demyelinating encephalomyelitis (ADEM) could be considered for the initial presentation but would be unlikely given her age and the time that elapsed between episodes.

\section{INVESTIGATIONS: DR. FLORENDO-CUMBERMACK}

She had the following blood work completed and all were found to be within normal limits: a complete blood count, urea, creatinine, electrolytes, calcium, magnesium, liver enzymes, albumin, random glucose, international normalized ratio, partial prothrombin time, creatinine kinase, troponin-T, thyroid stimulating hormone, serum angiotensin-converting enzyme (ACE), serum protein electrophoresis, antinuclear antibody, anti-ds DNA, anti-extractable nuclear antigen screen, anti- neuromyelitis optica (NMO) antibodies, and Lyme serology. Her vitamin B12 level was $198 \mathrm{pmol} / \mathrm{L}$. Her urinalysis was within normal limits.

Cerebrospinal fluid (CSF) testing revealed a glucose level of $3.7 \mathrm{mmol} / \mathrm{L}$, protein level of $577 \mathrm{mg} / \mathrm{L}$, and nucleated cell level of $1 \times 10^{6} / \mathrm{L}$ (80\% lymphocytes). CSF was negative for ACE and oligoclonal bands, and immunoglobulin $\mathrm{G}$ index was 0.6 (normal range). CSF culture was negative and herpes simplex virus 1 and 2 polymerase chain reaction tests were negative. CSF cytology was negative for malignancy and flow cytometry could not be performed as there were too few cells.

Chest X-ray was within normal limits. Electroencephalography revealed low voltage, diffuse delta activity over the left hemisphere, more prominent posteriorly without any seizures recorded.

\section{Neuroradiology: Dr. Sharma}

Initial CT scan of the head (Figure 1A) performed on admission revealed bilateral extensive posterior white matter hypodensities, more on the left, but extending across the corpus callosum, with some local volume expansion. A differential diagnosis of lymphoma versus high-grade glioma/glioblastoma was the primary consideration.

An MRI of the brain (Figure 1B-E) was performed a day later and again demonstrated extensive, predominantly posterior and left, centrum semiovale white matter signal change with a T1 hypointense and T2 hyperintense appearance and local mass effect. Interestingly, there was diffusion restriction along the rim with incomplete rim enhancements and lack of increased perfusion. The appearance was not typical for a high-grade malignancy or progressive multifocal leukoencephalopathy. Although a low-grade glioma could not be excluded, a tumefactive demyelinating lesion was favoured.

\section{DisCUSSION: DR. SELCHEN}

Although the initial radiographic considerations included glioma and lymphoma, these are not an easy fit with the clear recurrent history. Imaging as expected does not support a vascular etiology.

A demyelinating lesion is therefore a very strong possibilityspecifically, recurrent tumefactive demyelination. However, a lesion crossing the corpus callosum is distinctly unusual in this scenario. In spite of the "butterfly" type appearance, taking into account the history, demyelination remains the most likely diagnosis.

\section{NeUrosurgery: Dr. STEVen}

After obtaining informed consent, a framed-based CT-guided stereotactic biopsy was performed. A parietal biopsy site in the enhancing portion of the left-sided lesion was chosen. An intraoperative frozen section confirmed lesional tissue and the remainder of the specimen were sent for permanent pathological assessment. There were no perioperative complications.

\section{NeUropathology: Dr. ANG}

The biopsy was adequate in size, retrieving two samples, with the largest measuring approximately $5 \times 2 \times 2 \mathrm{~mm}$. The intraoperative consultation revealed reactive histiocytes and astrocytes consistent with a demyelinating process. Permanent sections and special stains concurred, finding a confluent area of demyelination in which axons were relatively spared. Within the demyelinated zone, macrophages contained phagocytosed myelin as demonstrated by Luxol Fast Blue staining. Inflammatory elements were mixed, dominated by macrophages, but accompanied by sparse lymphocytes, the majority being $\mathrm{T}$ cells expressing CD3 (with mixed CD4 and CD8 elements) (Figure 2).

The neuropathological diagnosis was acute demyelinating lesion.

\section{Discussion: Dr. SelChen}

The pathology is consistent with a demyelinating lesion, most likely tumefactive MS, as the gradual evolution of the symptoms would be against the possibility of NMO. However, despite a negative serum anti-NMO result, NMO spectrum disorder is still a consideration. Are there pathological ways of differentiating between tumefactive MS and NMO, and can the latter condition be excluded on pathological grounds? Clinically, I would very much favour tumefactive MS. 


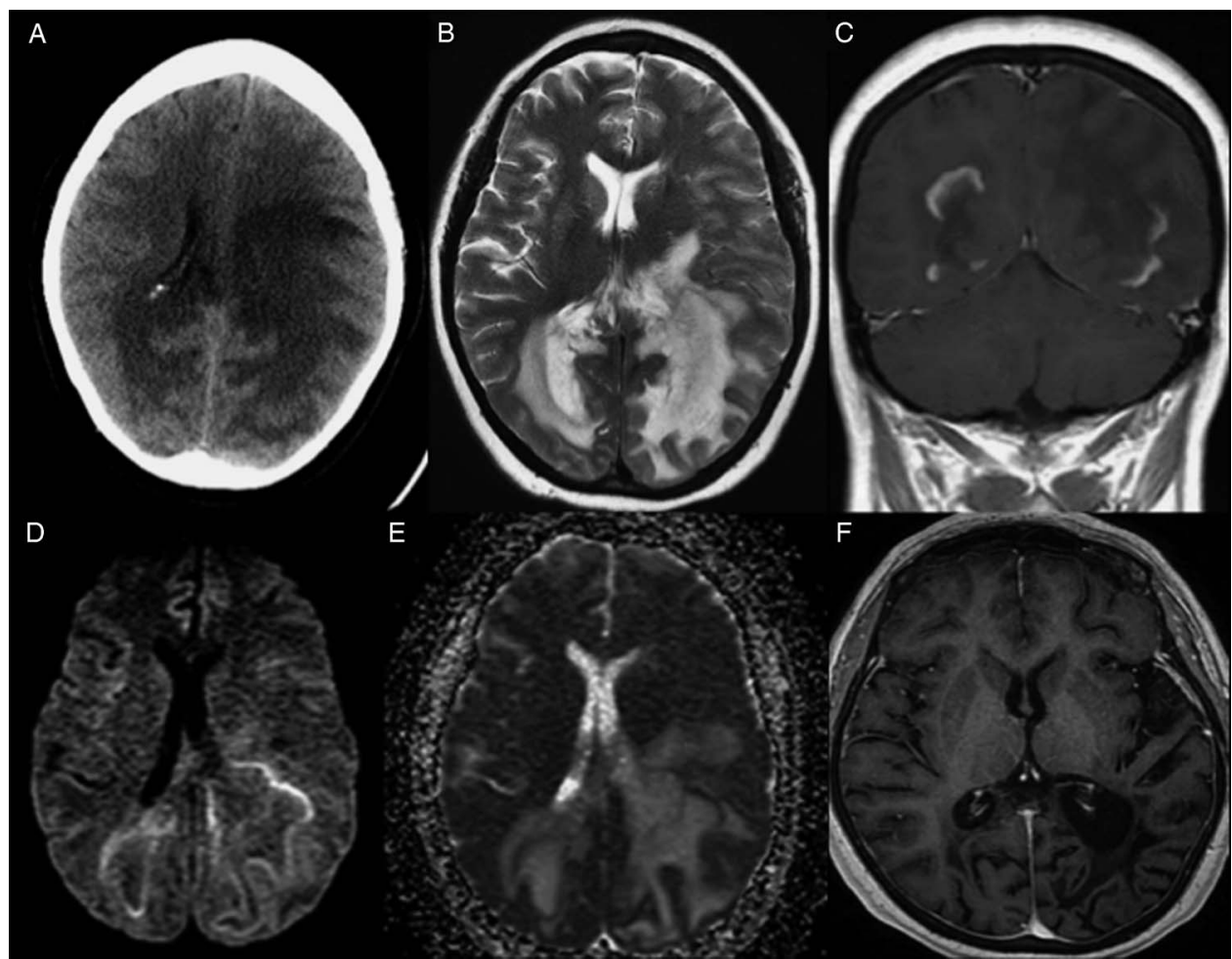

Figure 1: Computed tomography $(C T)$ and magnetic resonance imaging (MRI) from initial admission to our institution. (A) Initial CT on presentation to the emergency department shows large, predominately white matter hypodensities crossing midline but larger on the left, with some local mass effect. (B) T2-weighted axial MRI confirms findings with T2 hyperintense appearance. (C) Postgadolinium coronal T1 image shows incomplete ring enhancement around the affected areas. (D) Diffusion image and (E) corresponding apparent diffusion coefficient map show peripheral incomplete rim of restriction. $(F)$ Three-year follow-up T1-weighted MRI image shows evolution of the lesion.

\section{NeUropathology: Dr. ANG}

Tissue can be stained for aquaporin-4 antibodies (AQP4), but this immunohistochemical stain is not available in our facility and therefore the possibility of NMO could not be excluded.

\section{Patient's Progress: Dr. Florendo-Cumbermack}

Following stereotactic brain biopsy, the patient was treated with methylprednisolone $1 \mathrm{~g}$ for 5 days. There was noted improvement in her weakness, walking, language, and apraxia. She also received inpatient physiotherapy, speech, and occupational therapy.

Three months later, she was readmitted with behavioural issues and worsening gait. She was obsessed that her medication was a "poison" and became agitated when her daughter attempted to help. Her word generation was impaired for letter naming and often she just made nonsensical sounds. Immediate recall was impaired (1/20) despite multiple attempts, and delayed recall was nonexistent. She was unable to complete other formal testing for processing speed, executive function, or working memory. As previously described, her MRI showed worsening T2 abnormality in the left hemisphere with gadolinium enhancement. Once again, she was treated with a course of methylprednisolone, followed on this occasion by tapering doses of oral prednisone, and azathioprine was started. Symptoms improved and she was discharged home.
At her 6-month follow-up, she was noted to have a dense right homonymous hemianopia and, though improved, she was still quite aphasic. She did not tolerate the azathioprine because of vomiting and elevations in her liver enzymes; this was therefore discontinued after she was on it for about 3 months. She was subsequently lost to follow-up for more than a year but had no new symptoms during that time.

The patient was seen again 3 years after her second presentation. She remained seizure-free on levetiracetam $500 \mathrm{mg}$ twice daily. Though there had been an improvement overall, she continued to have significant cognitive deficits. She was independent with activities of daily living, but still had impairment of language and remained unable to read or write. She still had a dense right homonymous hemianopia. There was no weakness, but a right parietal drift was noted. She had apraxia mainly of the left hand to verbal command and there was some evidence of finger agnosia. She remained off immunosuppressive agents.

\section{Neuroradiology: Dr. Sharma}

A follow-up MRI performed 3 months after presentation revealed worsening of the white matter signal change with discontinuous, persistent, slightly thicker peripheral enhancement on the left, and some interval cystic change. This was considered slightly atypical for a demyelinating lesion. MRI repeated 9 days later revealed some improvement in the overall appearance with less enhancement. 
MRI performed 7 months after presentation showed further improvement with no residual enhancement. A long-term followup MRI scan (Figure 1F) 3 years after presentation revealed expected encephalomalacic changes with volume loss and ex vacuo dilation of the adjacent ventricle.

\section{Topic Review: Dr. Florendo-CuMbermack}

\section{Epidemiology}

Tumefactive demyelinating disease (TDD) continues to be referred to by many different names in the literature. TDD is characterized by clinical features that are atypical for MS, and brain imaging with a lesion greater than $2 \mathrm{~cm}$ with perilesional edema, mass effect, and open-ring gadolinium enhancement. ${ }^{1}$

A slight female predominance of 1.2:1 has been reported and the prevalence and incidence are estimated to be 1 to 2 per 1000 MS cases and 3 per 1,000,000 per year, respectively. ${ }^{1,2}$ Onset is typically in the third to fourth decades with a median age at onset of 37 years. ${ }^{1}$ Our patient's initial presentation was at age 51 years. TDD has been seen among the pediatric population as well.

\section{Clinical Presentation}

TDD was found to be the first demyelinating event in the majority $(61 \%)$ of patients in a study of 168 pathologically confirmed cases of central nervous system (CNS) demyelination. ${ }^{1}$ This was the case with our patient. In $29 \%$ of cases, it represented a relapse presentation in a patient already known to have relapsing remitting $\mathrm{MS}^{1}$ Most of these $(50 \%)$ patients had motor symptoms, and $43 \%$ had cognitive dysfunction, which included encephalopathy, memory dysfunction, aphasia, and apraxia. Sensory dysfunction, visual field defects (though homonymous hemianopia was rare), and seizures were also described. Our patient presented with all of these clinical features and, despite improvement in motor and sensory functioning, her cognition remained profoundly impaired. Additional clinical features described by Lucchinetti et $\mathrm{al}^{1}$ included cerebellar and brainstem findings.

\section{Immunopathogenesis}

There are no pathological features that distinguish the demyelination of TDD from that of $\mathrm{MS}^{3}$ Classic features of active demyelination include the presence of Creutzfeldt cells (reactive astrocytes undergoing "granular" mitoses) and foamy macrophages containing myelin. However, no clear immunopathological process for TDD has been elucidated.

\section{Radiological Features}

In some cases, the constellation of radiological features may be classic for the diagnosis of TDD. These features included lesion size greater than $2 \mathrm{~cm}$ on T2-weighted images, with edema and mass effect and incomplete ring enhancement with the ring opening towards either the cortical gray matter or the basal ganglia. ${ }^{1,3,4}$ Lesions were typically supratentorial and involved the frontal white matter most commonly, followed by the parietal subcortical white matter. ${ }^{1,3}$ A nonspecific butterfly configuration with involvement of the splenium of the corpus callosum was less commonly seen. ${ }^{1,3}$ Our patient had a contiguous butterfly lesion extending to the occipital, parietal, frontal, and temporal white matter. There was significant edema and mass effect.
The open-ring pattern of enhancement in our patient was towards the white matter.

Some authors suggest that other important imaging findings in TDD that may be helpful in distinguishing it from other conditions include unrestricted diffusion and evolving findings on serial magnetic resonance spectroscopy studies. ${ }^{3}$ This was in contrast to abscesses in which there was restricted diffusion, and to neoplasms, in which the magnetic resonance spectroscopy studies tend to have a stable pattern over time.

\section{Differential Diagnosis}

The differential diagnosis in TDD is extensive and includes glioblastoma multiforme, primary CNS lymphoma, brain metastases, brain abscess, and other causes of tumefactive demyelination besides MS. These include ADEM and combinations of ADEM with MS, which have also been reported in the literature. ${ }^{5}$ It is also important to distinguish TDD from other, more aggressive atypical variants of MS such as Balo concentric sclerosis and Marburg variant. ${ }^{3}$ Distinguishing TDD from glioblastoma multiforme or primary CNS lymphoma is important because both of the latter conditions may also initially respond to high-dose corticosteroids. Other causes of CNS demyelination include HIV; progressive multifocal leukoencephalopathy; postinfectious/ postvaccine encephalomyelitis; systemic lupus erythematosus; Sjögren's syndrome; Behcet's disease; NMO spectrum; drugs such as tacrolimus; and renal cell carcinoma, leukemia, myelodysplastic syndrome, and seminoma. ${ }^{3,6-8}$ Investigations should aim to exclude these conditions. Investigations that may be helpful include oligoclonal bands (positive in one-third of cases), immunoglobulin $\mathrm{G}$ index, antinuclear antibodies, anti-extractable nuclear antigen screen, lupus anticoagulants, anti-neutrophil cytoplasmic antibody, serum and CSF ACE, AQP4 antibodies, HIV serology, and brain biopsy. ${ }^{2}$ It is not possible to fully exclude NMO spectrum disorders without immunohistochemistry for AQP4 antibodies.

\section{Management}

As with other acute inflammatory demyelinating events, highdose corticosteroid therapy remains the mainstay of treatment, with $80 \%$ of patients reported to respond favourably. ${ }^{3}$ Plasma exchange is becoming popular for the $20 \%$ of patients who do not respond favourably to corticosteroids. ${ }^{3}$ Favourable outcomes from the use of rituximab has also been described in case reports. ${ }^{2}$ Hardy and Chataway ${ }^{2}$ have suggested that MS disease-modifying therapies such as mitoxantrone, interferon $\beta$, or natalizumab should be reserved for confirmed cases of $\mathrm{MS}^{3}$ Cyclophosphamide and intravenous immunoglobulins seem to be more successful for treating children with TDD. ${ }^{3,4}$ Decompressive craniectomy may be necessary in cases with sufficient mass effect. ${ }^{2}$

Our patient was treated with corticosteroids during her first presentation. This seems to have led to an 11-year remission. During her second presentation, her symptoms waxed and waned with improvement with high-dose corticosteroid treatment followed by worsening. Azathioprine was initiated with concurrent high-dose prednisone. This was discontinued after only 3 months because of hepatotoxicity and gastrointestinal upset with an uncertain, if any, immunosuppressive effect. Nonetheless, she was fortunate thereafter to experience a second long-standing remission. 

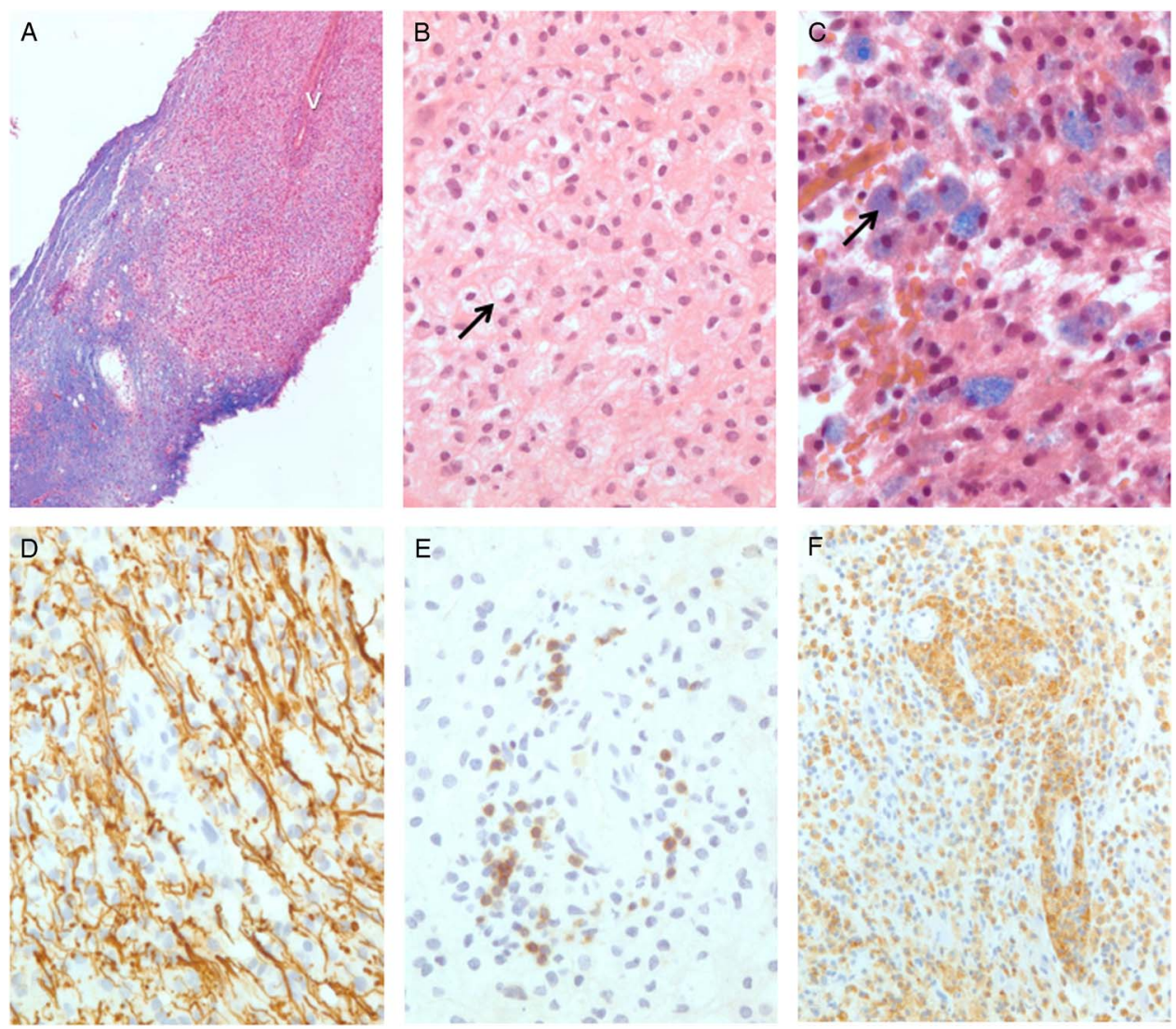

Figure 2: Photomicrographs of the left temporal-parietal biopsy. (A) Demyelination around small intraparenchymal vein, with normal myelin staining blue (v) (hematoxylin and Luxol Fast Blue). (B) Demyelinated regions are heavily infiltrated by foamy macrophages (arrow) (hematoxylin). (C) Foamy macrophages contain phagocytosed myelin (blue) (hematoxylin and Luxol Fast Blue). (D) Axons are relatively preserved in areas of demyelination (anti-neurofilament immunoperoxidase). (E) CD-3 positive lymphocytes are abundant (anti-CD3 immunoperoxidase). $(F)$ Macrophages are confirmed by CD68 expression (anti-CD68 immunoperoxidase).

\section{Prognosis}

Two-thirds of TDD patients developed definite MS, whereas $14 \%$, mainly older patients, had isolated events. ${ }^{1,3}$ In most patients, the second event was a typical MS demyelinating event; a second presentation with TDD, as in our patient, was a rare occurrence. $^{3}$ The median time to a second event was estimated to be 4.8 years. ${ }^{1}$ TDD should be distinguished from more aggressive forms of MS such as Marburg variant and Balo's concentric sclerosis where tumefactive lesions are also present, but the disease course is acute and progressive and can lead to death. TDD with disease duration of greater than 10 years was compared with an MS cohort of similar duration and found to have a better Expanded Disability Status Scale score. ${ }^{1}$ Lesions greater than $5 \mathrm{~cm}$ were however associated with slightly worse Expanded Disability Status Scale scores. ${ }^{1}$

\section{CONCLUSION}

This case report highlights many typical as well as some atypical features of TDD. It also highlights the diagnostic dilemma that the neurologist and neuroradiologist can face with TDD, often leading to a need for one or more brain biopsies.
The recurrence after ten years in this patient suggests that follow-up of patients with TDD should be prolonged even if no new clinical events occur.

\section{DisClosures}

SM has the served as a consultant/advisor and received consulting fees from Biogen IDEC, EMD Serono, Novartis, and Genzyme; received an IIT grant from Biogen IDEC; and served on a speaker's bureau and received honoraria from Biogen IDEC, EMD Serono, and Novartis. CC is a fellow and received funding for a fellowship through Dr. Paul O'Connor; has served as a consultant and received consultant fees from EMD Serono; and received funding for travel to the ECTRIMS Conference from EMD Serono. JB has served on a speaker's bureau and received honoraria from UCB and EISAI and served on an advisory board and received honoraria from Sunovion. MK has been a grant recipient and consultant and received grants and consulting fees from Biogen IDEC, Genzyme, and Novartis; been a researcher for and received research grants from Bayer, Sanofi, Teva, and CIHR; and has received operational grants from the Society of Canada. AF-C, DS, MS, DS, LCA, and RH have nothing to disclose. 


\section{REFERENCES}

1. Lucchinetti CF, Gavrilova RH, Metz I, Parisi JE, Scheithauer BW, Weigand $\mathrm{S}$, et al. Clinical and radiological spectrum of pathologically confirmed tumefactive multiple sclerosis. Brain. 2008;131:1759-75.

2. Nilsson P, Larsson EM, Kahlon B, Nordström CH, Norrving B. Tumefactive demyelinating disease treated with decompressive craniectomy. Eur J Neurol. 2009 May;16(5):639-42. doi: 10.1111/j.1468-1331.2009.02547.x. Epub 2009 Mar 20.

3. Hardy TA, Chataway J. Tumefactive demyelination: and approach to diagnosis and management. J Neurol Neurosurg Psychiatry. 2013;84:1047-53.

4. Turatti M, Gajofatto A, Bianchi MR, Ferrari S, Monaco S, Benedetti MD. Benign course of tumour-like multiple sclerosis. Report of five cases and literature review. J Neurol Sci. 2013;324:156-62.
5. Koshihara H, Oguchi K, Takei Y, Kitazawa K, Higuchi K, Ohara S. Meningeal inflammation and demyelination in a patient clinically diagnosed with acute disseminated encephalomyelitis. J Neurol Sci. 2014;346:323-7.

6. Rahmlow MR, Kantarci O. Fulminant demyelinating disease. Neurohospitalits. 2013;3:81-91.

7. Hane A, Bargetzi M, Hewer E, Bruehlmeier M, Khamis A, Roelcke U. Recurrent tumefactive demyelination without evidence of multiple sclerosis or brain tumour. J Neurol. 2011;258:318-20.

8. Kepes JJ. Large focal tumor-like demyelinating lesions of the brain: indeterminate entity between multiple sclerosis and acute disseminated encephalomyelitis? A study of 31 patients. Ann Neurol. 1993;33:18-27. 